\title{
Aciotis, Acisanthera, Marcetia e Pterolepis (Melastomeae-Melastomataceae) no estado do Rio de Janeiro
}

\author{
Aciotis, Acisanthera, Marcetia and Pterolepis (Melastomeae-Melastomataceae) \\ in Rio de Janeiro state
}

Paulo José Fernandes Guimarães ${ }^{1,4}$ \& Marcus Felippe Oliveira da Silva ${ }^{2,3}$

\begin{abstract}
Resumo
É apresentado o tratamento taxonômico para os gêneros Aciotis, Acisanthera, Marcetia e Pterolepis. (Melastomeae, Melastomataceae) no estado do Rio de Janeiro. Cada um dos quatro gêneros apresentados está representado por uma única espécie no estado: Aciotis paludosa, Acisanthera variabilis, Marcetia taxifolia e Pterolepis glomerata. Com este estudo se atualizou a lista de espécies para a flora do estado do Rio de Janeiro, removendo seis espécies equivocadamente listadas. Um tratamento taxonômico à parte está sendo providenciado para as espécies de Tibouchina sensu lato, que serão segregadas entre os gêneros Chaetogastra e Pleroma (que inclui Itatiaia). O tratamento taxonômico apresentado para os gêneros inclui chave de identificação, descrições, comentários taxonômicos, informações sobre distribuição geográfica, categorização de risco de extinção segundo os critérios da IUCN, lista de materiais examinados e ilustrações. Palavras-chave: taxonomia, flora, região sudeste do Brasil.
\end{abstract}

\begin{abstract}
A taxonomic survey of the genera Aciotis, Acisanthera, Marcetia and Pterolepis (Melastomeae, Melastomataceae) in Rio de Janeiro state is presented here. The four genera were studied and they are represented in Rio de Janeiro by one species each: Aciotis paludosa, Acisanthera variabilis, Marcetia taxifolia and Pterolepis glomerata. In this paper, the Rio de Janeiro species list has been updated, removing six species mistakenly listed. Tibouchina sensu lato will be presented in a separate paper, with its species segregated between Chaetogastra and Pleroma (that includes Itatiaia). The present taxonomic treatment includes an identification key, descriptions, comments on taxonomy, data on geographic distribution, categorization of extinction risk under IUCN criteria, list of examined material and illustrations.
\end{abstract}

Key words: Taxonomy, floristics, Southeastern Brazil.

\section{Introdução}

Melastomataceae Juss. é uma das maiores famílias dentre as Angiospermas (Clausing \& Renner 2001) e apesar de estar distribuída por toda a região tropical (Renner 1993) apresenta maior número de espécies no neotrópico (Almeda 2009).

O Brasil possui nove tribos (Renner 1993; Clausing \& Renner 2001; Penneys et al. 2010; Michelangeli et al. 2013), 67 gêneros e 1.329 espécies (Baumgratz et al. 2014) e se destaca na região neotropical como um dos países com maior número de espécies de Melastomataceae, à frente da Argentina com 27 sp., (Peralta 2002), Colômbia com ca. de 900 sp. (Mendoza \& Ramirez 2006), Guiana com 427 sp. (Berry et al. 2001), Equador com 553 sp. (Freire-Fierro et al. 2002) e Venezuela com 650 sp.(Michelangeli \& Cotton 2008).

Dentre as tribos, Melastomeae se destaca por ser uma das poucas com distribuição pantropical e apresentar cerca de 870 espécies distribuídas em 47 gêneros (Michelangeli et al. 2013). No Brasil são registrados 22 gêneros e 290 espécies, que a

\footnotetext{
'Instituto de Pesquisas Jardim Botânico do Rio de Janeiro, DIPEQ, R. Pacheco Leão 915, 22460-030, Rio de Janeiro, RJ, Brasil.

${ }^{2}$ Secretaria Estadual de Educação, Regional Norte Fluminense, R. $1^{\circ}$ de Maio 9, 28035145, Campos dos Goytacazes, RJ, Brasil.

${ }^{3}$ Escola Nacional de Botânica Tropical, ENBT-JBRJ, R. Pacheco Leão 2040 , 22460-036, Rio de Janeiro, RJ, Brasil.

${ }^{4}$ Autor para correspondência: paulojose.guimaraes@gmail.com
} 
colocam como a tribo com maior número de gêneros e a segunda com maior número de espécies, uma vez que Miconieae apresenta ca. de 600 espécies (Baumgratz et al. 2014).

Melastomeae pode ser reconhecida com base nas seguintes características: (1) estames com pedoconectivo (pouco desenvolvido ou mesmo reduzido em alguns poucos gêneros), (2) apêndices, se presentes, ventrais e dispostos aos pares, (3) Frutos capsulares com sementes cocleadas e embriões curvos (Michelangeli et al. 2013).

Entretanto, as espécies da tribo Melastomeae (sensu Clausing \& Renner 2001) ocorrentes na região neotropical não se mostram como um grupo monofilético, sendo subdivididas em três clados: "Rhexieae", "Marcetia alliance" e "Core Melastomeae", caracterizados com base na morfologia dos estames, ovário e sementes (Michelangeli et al. 2013).

Neste estudo foram registrados gêneros pertencentes aos dois últimos clados, cujas espécies alcançam a região sudeste do Brasil. Estes podem ser reconhecidos com base nas sinapomorfias reveladas por Michelangeli et al. (2013), a saber: "Marcetia alliance" (1) flores tetrâmeras (raramente pentâmeras), (2) ápice do ovário glabro ou coberto apenas por tricomas glandulares, (3) sementes cocleadas, ovaladas ou lacrimiformes com superfície foveolada, com exceção de Siphanthera (Whiffin \& Tomb 1972; Almeda \& Robinson 2011) e para "Core Melastomeae" (1) flores pentâmeras ou tetrâmeras (2) ápice do ovário recoberto por tricomas, (3) sementes cocleadas com tuberculos ou papilas (Michelangeli et al. 2013).

Entretanto, novas proposições taxonômicas baseadas em inferência filogenética (Michelangeli et al. 2013) deverão ser apresentadas em breve para Melastomeae sensu stricto e os demais clados (D. Penneys com. pess.) assim, adotouse aqui uma circunscrição mais ampla, segundo Renner (1993). No estado do Rio de Janeiro, Melastomeae está representada pelos gêneros Aciotis D. Don, Acisanthera P.Browne, Marcetia DC., Pterolepis (DC.) Miq. e Tibouchina Aubl. sensu lato (Baumgratz et al. 2014).

Além disso, as espécies de Tibouchina Aubl. sensu lato ocorrentes no estado serão futuramente transferidas para os gêneros Chaetogastra DC. e Pleroma D. Don (P.J.F. Guimarães \& F.A. Michelangeli, em preparação), enquanto o gênero monotípico Itatiaia Ule, endêmico ao estado, será futuramente transferido para Pleroma (M.F.Oliveira-da-Silva et al. 2014).
É aqui apresentado o tratamento taxonômico parcial dos gêneros de Melastomeae para o Estado do Rio de Janeiro, exceto para Tibouchina sensu lato, que é mais diverso, e será tratado em um trabalho à parte (P.J.F. Guimarães, em preparação).

São apresentadas chave de identificação, descrições dos táxons, comentários taxonômicos, informações sobre habitat, distribuição geográfica, listagens de material examinado e ilustrações.

\section{Material e Métodos}

As exsicatas analisadas neste trabalho são provenientes dos herbários GUA, HB, R e RB (acrônimos segundo Thiers continuously updated). Embora esteja citada apenas uma amostra por município do estado do Rio de Janeiro, no final do trabalho é fornecida a lista completa de coletores, incluindo todas as exsicatas analisadas para o tratamento taxonômico.

Táxons infragenéricos (seções) e infraespecíficos (variedades) não foram considerados neste trabalho, pois não possuem uma delimitação clara. São apresentadas descrições para a tribo e para os gêneros incluídos no tratamento, bem como para as espécies.

A terminologia adotada para morfologia geral segue Radford et al. (1974), exceto no caso do indumento onde se segue Hickey \& King (2000), e frutos onde se segue Baumgratz (1985). Para a identificação dos gêneros de Melastomataceae no Brasil utilizamos a chave de Goldenberg et al. (2012).

A classificação fitogeográfica segue Veloso et al. (1991), e informações sobre distribuição geográfica dos táxons foram também obtidas em Baumgratz et al. (2014), Freire-Fierro (2002), Kriebel (2014), Kriebel \&Almeda (2013), Guimarães (2009) e Renner (1994).

Dados de floração e frutificação para as espécies se baseiam apenas nas informações contidas em etiquetas de exsicatas provenientes do estado do Rio de Janeiro. Todas as espécies registradas neste estudo foram avaliadas e categorizadas segundo os critérios de conservação da IUCN como "pouco preocupante" (LC).

\section{Resultados e Discussão}

A tribo Melastomeae inclui cerca de 570 espécies na América do Sul (Michelangeli et al. 2013). No Brasil são listadas 292 espécies (Core Melastomeae: 178 sp; Marcetia alliance: $112 \mathrm{sp}$; Comoliopsis Wurdack:1sp., tem posição incerta), incluídas em 22 gêneros (Baumgratz 2014a-1; Baumgratz \& Lima 2014; Baumgratz \& Rosa 2014; Kriebel 2014; Martins \& Rodrigues 2014; 
Romero 2014a-d; Goldenberg 2014; Guimarães 2014), enquanto que para o estado do Rio de Janeiro são registrados os gêneros Aciotis, Acisanthera, Marcetia e Pterolepis, representados por apenas 1 espécie cada e também Tibouchina sensu lato (incluindo os gêneros Chaetogastra, e Pleroma), com cerca de 50 espécies. As espécies de Aciotis, Acisanthera, Marcetia e Pterolepis que ocorrem no estado do Rio de Janeiro são pouco diversos em comparação com Tibouchina, uma vez que a Mata Atlântica é uma região de elevada diversidade para este último gênero (Michelangeli et al. 2013), enquanto os demais possuem maior diversidade de espécies na Colômbia (Freiro-Fierro 2002) e na região central do Brasil (Renner 1993; Santos et al. 2013).

A baixa diversidade destes gêneros no estado do Rio de Janeiro é definida pelo fato de que estes são mais diversos no Cerrado em áreas abertas, em campo de altitude e rupestre (Freire-Fierro 2002; Kriebel 2008; Renner 1994; Santos et al. 2013), sendo este bioma ausente no estado.

Aciotis acuminifolia (Mart. ex DC) Triana, Aciotis ferreirana Brade, Aciotis polystachya (Bonpl.) Triana, Acisanthera glazioviana Cogn., Acisanthera quadrata Pers. e Marcetia bracteolaris (Schrank \& Mart. ex DC.) O. Berg ex Cogn. são listadas para a flora do estado do Rio de Janeiro (Baumgratz \& Rosa 2014; Kriebel 2014; Martins \& Rodrigues 2014). Esta informação não foi sustentada com base na morfologia e distribuição geográfica dos táxons durante as análises e devem estar relacionadas com identificações incorretas ou informações equivocadas em etiquetas de coleta.

Recentemente, alguns trabalhos florísticos com Melastomataceae foram realizados no estado do Rio de Janeiro, mas estes na maioria das vezes são restritos às unidades de conservação (Baumgratz et al. 2006; Baumgratz et al. 2007; Barberena et al. 2008; Baumgratz \& Souza 2011; Silva et al. 2013) sendo mais raros os tratamentos taxonômicos para gêneros (Baumgratz et al. 2004; Chiavegatto \& Baumgratz 2008; Silva \& Baumgratz 2008). Pereira (1960-1961) realizou um trabalho com os gêneros de Tibouchineae (=Melastomeae) para o estado da Guanabara, hoje, município do Rio de Janeiro, mas este é o primeiro estudo taxonômico com os gêneros de Melastomeae para o estado do Rio de Janeiro e com ele se espera contribuir para o conhecimento da diversidade e conservação de Melastomataceae neste estado.

\section{Tratamento taxonômico \\ Tribo Melastomeae}

Flores tetrameras ou pentâmeras, cálice simples sem lacínias externas, estames dimorfos ou isomorfos, pedoconectivo (conectivo ventralmente prolongado abaixo da antera) presente ou reduzido ou ausente, apêndices ventrais, bilobados, filiformes ou ausentes. Ovário 2-5 locular, ápice com indumento seríceo, glandular ou glabro. Frutos capsulares ou raramente bacáceos, sementes numerosas cocleadas, ovadas ou raramente lacrimiformes, testa com tubérculos, alvéolos ou aréolas.

\section{Chave para identificação de gêneros e espécies de Melastomeae no estado do Rio de Janeiro}

1. Hipanto com projeções vascularizadas e ramificadas ...................................................Pterolepis glomerata

1'. Hipanto sem projeções vascularizadas e ramificadas........................................................................................

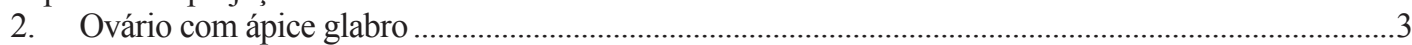

3. Anteras falciformes com pedoconectivo conspícuo, ca. $8 \mathrm{~mm}$ compr., geniculado com apêndices ventrais bilobados ..................................................................................... Acisanthera variabilis

3'. Anteras eretas ou levemente arqueadas sem pedoconetivo (ou com até $0,1 \mathrm{~mm}$ de compr.) e sem apêndices ventrais.

4. Ervas; lâmina foliar 0,5-1 cm compr., 5 nervuras, margem plana; anteras ca. $1 \mathrm{~mm}$ compr. Aciotis paludosa

4. Subarbustos a arbustos; lâmina foliar $\leq 0,5 \mathrm{~cm}$ compr., (1)-3 nervuras, margem revoluta; antera ca. $4 \mathrm{~mm}$ compr.............................................................................. Marcetia taxifolia

2'. Ovário com ápice recoberto por indumento seríceo ou glandular no ápice ...............................................

5. Arbustos ou arvoretas, raramente ervas; anteras com coloração roxa, lilás, creme ou brancas raramente amarelas; conectivo com apêndices ventrais, com glândulas pediceladas ou glabros; filetes com indumento variado ou glabros; lacínias do cálice caducas no fruto, raramente persitentes ...............................................................................................................Pleroma

5'. Ervas ou subarbustos; anteras com coloração total ou parcialmente amarelas; conectivo com apêndices ventrais, glabros; filetes glabros; lacínias do cálice persistentes no fruto. 
1. Aciotis D. Don, Mem. Wern. Nat. Hist. Soc. 4: 283, 300. 1823.

Ervas até $1 \mathrm{~m}$ alt., anuais ou perenes, estoloníferas ou não; ramos quadrangulares, subalados a alados, glabros ou com tricomas glandulares ou não. Folhas planas. Flores 4-meras; estames 8 , isomorfos, anteras orbiculares ou oblongas, conectivo curtamente ou não prolongado abaixo das tecas, inapendiculado; ovário semiínfero, 2(-3)-locular, glabro. Fruto capsular ou bacáceo.

Aciotis é um gênero caracterizado pelo porte herbáceo, flores tetrâmeras pequenas, anteras com pedoconectivo curto de 0,1 a $0,6 \mathrm{~mm}$ de comprimento, ovário geralmente bilocular, frutos secos ou bacáceos e indeiscentes em algumas espécies (Freire-Fierro 2002). São reconhecidas atualmente 13 espécies de distribuição neotropical (Freire-Fierro 2002), todas ocorrem no Brasil (Baumgratz \& Rosa 2014). No estado do Rio de Janeiro foi reconhecida apenas A. paludosa.

1.1 Aciotis paludosa (Mart. ex DC.) Triana, Trans. Linn. Soc. London 28: 51. 1871. Spennera paludosa Martius ex DC., Prodr. 3: 115. 1828.

Fig 1a-d

Ervas ca. $30 \mathrm{~cm}$ alt.; ramos e pecíolos quadrangulares, alados, indumento dos ramos e pecíolos densamente setuloso e nos eixos das inflorescências setuloso-glanduloso. Folhas com pecíolos 2-2,5 cm compr; lâmina $6,5-7 \mathrm{~cm} \times$ 3,5-4 cm, oblongo-lanceolada, base atenuada, ápice obtuso, margem plana, serreado-ciliada, face adaxial glabra a esparsamente setosa, face abaxial glabra a pubérula, indumento avermelhado mais adensado sobre as nervuras, nervuras 5-basais. Inflorescências cimosas bíparas, raro uníparas, terminais ca. $4 \mathrm{~cm}$ compr., brácteas $2-4 \mathrm{~mm}$ compr., oval-lanceoladas; bractéolas ovais, ápice agudo. Flores 4-meras; hipanto ca. 2,5 mm compr., urceolado, esparsamente setoso-glanduloso, lácinias do cálice ovais, ápice agudo; pétalas 2,5 $\times$ $1 \mathrm{~mm}$, alvacentas a purpúreas, ovadas; estames 8 , isomorfos, com filetes ca. $2 \mathrm{~mm}$ compr., glabros, pedoconectivos ausentes até ca. $0,1 \mathrm{~mm}$ compr., inapendiculados, anteras ca. $1 \mathrm{~mm}$ compr., eretas, oblongas; ovário glabro, estilete 3-3,5 mm compr. Bacáceo ca. 3,5 mm compr.

Material selecionado: Magé, 11.I.2011, fl., B. De-Polli 18 (RB). Nova Iguaçu, 25.X.2002, fl. e fr., M.G. Bovini 2217 (RB). Petrópolis, I.1940, fl., B. Lutz 1563 (RB).
Rio de Janeiro, 5.II.1972, fr., L. Emygdio et al. 3086 (R). Silva Jardim, 3.II.1995, fl. e fr., J.M.A. Braga 1900 (RB). Teresópolis, 10.XII.1891, fl. e fr., E. Ule (R 166536).

Aciotis paludosa é endêmica do Brasil, mas apresenta ampla distribuição ocorrendo nos estados da Bahia, Maranhão, Pernambuco, Distrito Federal, Mato Grosso, Espírito Santo, Minas Gerais, Rio de Janeiro, São Paulo e Paraná (Baumgratz \& Rosa 2014). Tem preferência por locais perturbados, geralmente associados com água parada (Freire-Fierro 2002) e no Rio de Janeiro ocorre em Floresta Ombrófila Densa em elevações que podem variar desde os 40 até os 700 metros ao nível do mar.

Aciotis paludosa possui folhas com ápice obtuso, com 5 nervuras, e recobertas por indumento avermelhado, esparsa a moderadamente distribuído em ambas as faces da lâmina. A espécie mais próxima é $A$. acuminifolia (Mart. ex DC) Triana, que ocorre nas Antilhas e em quase toda a América do Sul, com exceção de Argentina, Chile, Paraguai e Uruguai (Freire-Fierro 2002) e no Brasil nos estados do Acre, Amapá, Amazonas, Maranhão, Mato Grosso, Pará e Rondônia. Aciotis acuminifolia difere de A. paludosa pelas folhas com ápice acuminado, trinervadas e geralmente glabras. (Freire-Fierro 2002).

Aciotis acuminifolia foi relacionada para o estado do Rio de Janeiro (Baumgratz \& Rosa 2014) com base no espécime E. Pereira 629 (R, RB) que apresenta uma menor densidade de tricomas nas folhas e ramos, além da ocorrência quase que imperceptível de tricomas glandulares no hipanto e inflorescências. Esta coleta possui procedência da Serra dos Órgãos, município de Guapimirim, e consta na lista de material examinado na revisão do gênero como Aciotis acuminifolia (Freire-Fierro 2002). Contraditoriamente, o estado do Rio de Janeiro foi omitido da distribuição geográfica de A. acuminifolia por Freire-Fierro (2002). Após a análise das coleções e confronto com a distribuição geográfica das duas espécies, optou-se aqui por considerar o espécime E. Pereira 629 dentro da variação morfológica observada para A. paludosa.

Os espécimes A.F.M. Glaziou 9822 (P) e 9877 (R), também referenciadas para o estado nas etiquetas representam respectivamente Aciotis ferreirana Brade e A. polystachya (Bonpl.) Triana, espécies que no território brasileiro são restritas à Amazônia (Freire-Fierro 2002). Estas são prováveis coletas de C.A.W. Schwacke distribuídas 


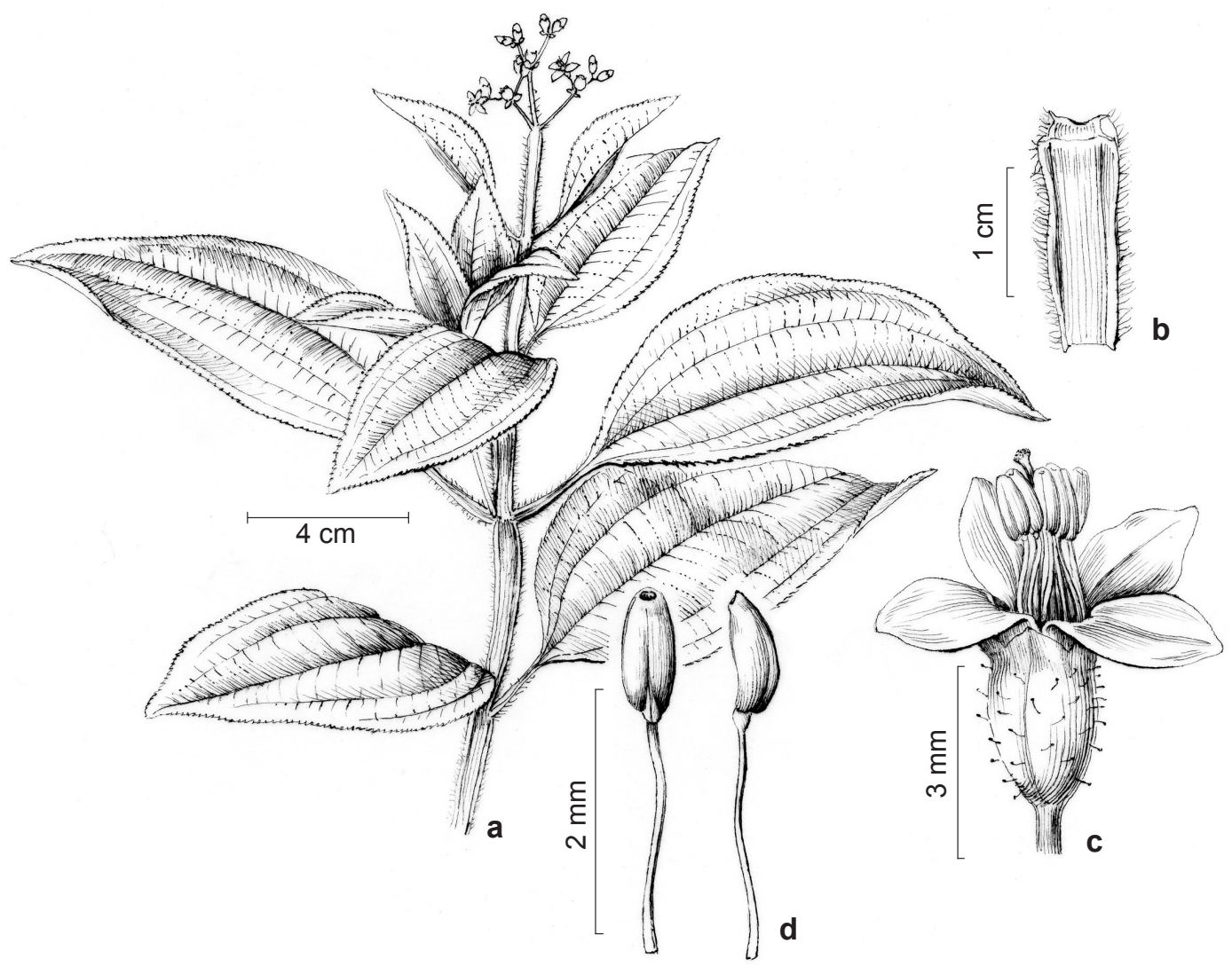

Figura 1 - a-d. Aciotis paludosa - a. hábito. b. detalhe do ramo; c. flor em antese. d. estames de ambos os ciclos em vista ventral e lateral (J.M.A. Braga 1900).

Figure 1 - a-d. Aciotis paludosa -a. habit. b. detail of branches; c. flower at anthesis; d. stamens of both cycles in frontal and lateral view (J.M.A. Braga 1900).

por A.F.M. Glaziou sob seu próprio nome (<plants. jstor.org> 2014). Schwacke e Glaziou têm o mesmo número para estas plantas. Como as coleções de Glaziou muitas vezes apresentam informações equivocadas em suas etiquetas (Wurdack 1970; Freire-Fierro 2002; Romero \& Versiane 2014) e até hoje não existem coletas conhecidas destas espécies fora da região amazônica, optou-se por não incluir estes espécimes no trabalho.

Aciotis paludosa foi coletada no estado do Rio de Janeiro com flores entres os meses de outubro e fevereiro, e com frutos entre janeiro e fevereiro. O status de conservação é pouco preocupante (LC), por ser uma espécie de ampla distribuição, que possui um número significativo de coletas, várias delas recentes. Além disso, $A$. paludosa ocorre em áreas de conservação como a Reserva Biológica de Poço das Antas (Baumgratz et al. 2006).
2. Acisanthera P. Browne, Civ. Nat. Hist. Jamaica 217. 1756.

Ervas ou subarbustos até 5-60 cm alt., anuais ou perenes; ramos quadrangulares, alados. Folhas planas. Flores 5-meras; estames (4-)8-10, alternadamente dimorfos, algumas vezes com um dos ciclos imperfeito, anteras oblongas, lineares, lanceoladas, subuladas ou truncadas, conectivos dos estames antepétalos pouco prolongados abaixo das tecas, com apêndices ventralmente bilobados, conectivos dos estames antessépalos longamente prolongados, com apêndices ventralmente bilobados, bífidos ou calcarados; ovário súpero ou semi-infero, 2-4-locular, glabro. Fruto capsular, revestido pelo hipanto persistente.

Acisanthera é reconhecido por suas flores com estames dimorfos dispostos em dois ciclos, anteras subuladas e presença de pedoconectivo com apêndices bilobados ventrais. É próximo a 
Tibouchina sensu lato, do qual difere por apresentar o ovário glabro, exceto por $A$. tetraptera que tem tricomas glandulares esparsamente distribuídos no ápice ovário, e sementes com superfície foveolada do tipo microlicióide (Whiffin \& Tomb 1972). São reconhecidas atualmente 18 espécies para o gênero, todas com distribuição neotropical (Kriebel \& Almeda 2013). As espécies de Acisanthera encontram-se distribuídas por todo o território brasileiro, exceto nos estados de Alagoas, Rondônia, Sergipe e Tocantins, onde ocorrem 13 espécies, 4 delas endêmicas. (Kriebel 2014). No estado do Rio de Janeiro foi reconhecida apenas Acisanthera variabilis (DC.) Triana.

As espécies de Acisanthera ocorrem geralmente em ambientes associados com água e são muitas vezes confundidas com espécies de Tibouchina de pequeno porte (como Tibouchina sebastianopolitana), pertencentes ao grupo que será transferido para Chaetogastra.

Na lista de espécies da flora do Brasil (Kriebel 2014) são relacionadas para o estado do Rio de Janeiro Acisanthera glazioviana Cogn., A. quadrata Pers.e A. variabilis (DC.) Triana. Acisanthera quadrata é quase limitada a regiões ao norte da Amazônia e caracterizada pela presença de um poro amplo na antera com $0,38 \pm 0,03 \mathrm{~mm}$ de diâmetro e flores axilares solitárias (Kriebel \& Almeda 2013). Acisanthera glazioviana está representada pela coleta de A.F.M. Glaziou de número 10769 realizada no estado do Rio de Janeiro em Palmital na "route du Picú", em companhia de L. Netto e C.A.W. Schwacke. A localização de Palmital é imprecisa, uma vez que Glaziou ora a cita para o Rio de Janeiro (Aranha Filho 2011) ora para Minas Gerais (Barroso 1958) nas coletas desta viagem. Assim como J.J. Triana, nós estamos determinando este material de A.F.M. Glaziou 10769 como A. variabilis. Como os espécimes encontrados nas coleções examinadas para o estado do Rio de Janeiro se enquadram na descrição de Acisanthera variabilis, esta espécie foi a única tratada neste trabalho.

2.1 Acisanthera variabilis (DC.) Triana, Trans Linn. Soc. London 28: 34. t. 11. f. 18 g. 1871. Microlicia variabilis Mart. ex DC., Prod. 3: 118. 1828.

Fig 2a-c

Subarbustos ca. $80 \mathrm{~cm}$ alt.; ramos quadrangulares, subalados, indumento dos ramos, pecíolos e eixos das inflorescências densamente hirsuto-glanduloso. Folhas com pecíolos 1-1,5 mm compr; lâmina 0,9-2 × 0,7-1,5 cm, cordada ou oval, base cordada, ápice agudo, margem plana, serreada, face adaxial glabra ou esparsa a moderadamente hirsuto-glandulosa, face abaxial glabra ou esparsamente hirsuto-glandulosa sobre as nervuras, nervuras 5-basais. Inflorescências em tirsóides ou cimeiras paucifloras, terminais 11-27 cm compr., brácteas ca. $4 \mathrm{~mm}$ compr., ovais, bractéolas reduzidas. Flores 5-meras; hipanto ca. $3 \mathrm{~mm}$ compr., oblongo, moderadamente hirsuto-glanduloso, lacínias do cálice lineares, ápice agudo; pétalas $6 \times 4 \mathrm{~mm}$, róseas, obovais; estames 10, dimorfos, antepétalos com filetes ca. 3,5 mm compr., glabros, pedoconectivos ca. 0,8 $\mathrm{mm}$ compr., apêndice ventral bilobado, anteras ca. 3,5 mm compr., falciformes, subuladas; antessépalos com filetes ca. $4 \mathrm{~mm}$ compr., glabros, pedoconectivos ca. $2,5 \mathrm{~mm}$ compr., apêndice ventral conspicuamente bilobado, anteras ca. $4 \mathrm{~mm}$ compr., subuladas; ovário glabro, estilete ca. $5 \mathrm{~mm}$ compr. Velatídio ca. $4 \mathrm{~mm}$ compr.

Material selecionado: Itatiaia, 22.I.1942, fl. e fr., $W$. D. de Barros 561 (RB). Nova Iguaçu, fl., 14.I.2002, M.G. Bovini 2112 (RB). Petrópolis, 25.I.1969, fl. e fr., D. Sucre 4491 (RB). Teresópolis, 11.III.1983, fl. e fr., A. Sampaio 1612 (R).

Acisanthera variabilis é endêmica do Brasil, mas apresenta ampla distribuição, com ocorrência nos estados da Bahia, Paraíba, Piauí, Distrito Federal, Goiás, Mato Grosso, Espírito Santo, Minas Gerais, Rio de Janeiro, São Paulo, Paraná e Santa Catarina (Meyer \& Goldenberg 2012; Kriebel 2014). No estado do Rio de Janeiro é geralmente encontrada em Floresta Ombrófila Densa, em pastagens e áreas alteradas, desde o nível do mar até elevações acima de 1000 metros de altitude.

Apresenta tricomas glandulares na face adaxial da lâmina foliar, ovário com ápice glabro e conectivos dos estames ventralmente bilobados, características que em conjunto permitem reconhecer esta espécie. Acisanthera variabilis é uma espécie bastante polimórfica em relação à morfologia das folhas e dos estames, o que torna obscura a sua delimitação em relação a espécies próximas (Kriebel 2008; Meyer \& Goldenberg 2012; Kriebel \& Almeda 2013).

Acisanthera variabilis foi coletada no estado do Rio de Janeiro com flores em setembro e flores e frutos de dezembro a abril. O status de conservação é pouco preocupante (LC), por ser uma espécie de ampla distribuição e que possui um número significativo de coletas, várias delas recentes. 


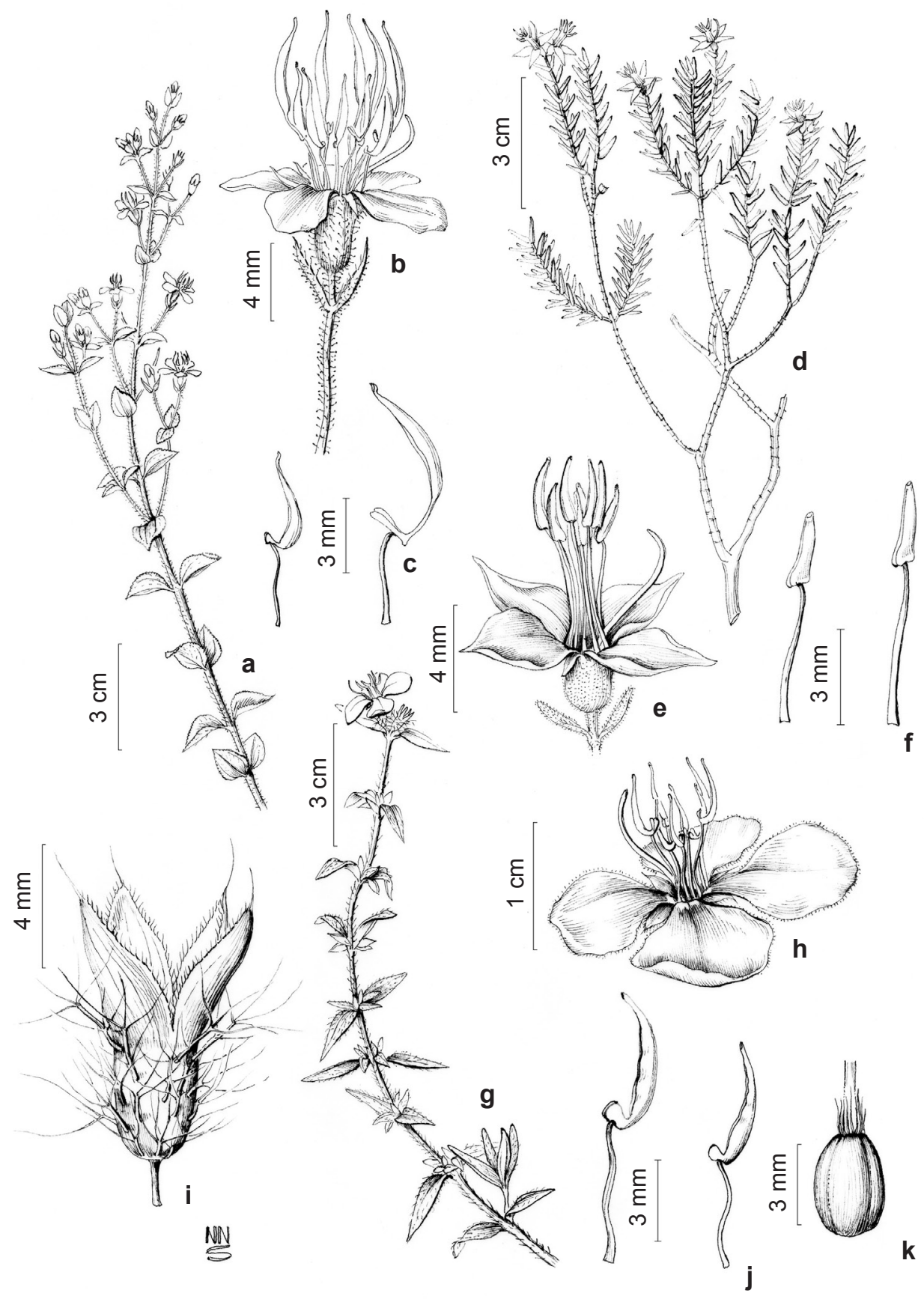

Figura 2 - a-c. Acisanthera variabilis - a. hábito; b. flor em antese completa com bractéolas na base do pedicelo; c. estame de ambos os ciclos em vista lateral (Burnet (RB 35219)). d-f. Marcetia taxifolia - d. hábito; e. flor em antese com bractéolas dispostas na base do pedicelo; f. estame de ambos os ciclos em vista lateral (J.F.A. Baumgratz 799). g-k. Pterolepis glomerata - g. hábito; h. flor em antese; i. fruto capsular revestido por projeções vascularizadas e tricomas na epiderme; j. estames de ambos os ciclos em vista lateral; k. ovário sem o estilete, revestido por tricomas no ápice (S. Araújo 493).

Figure 2 - a-c. Acisanthera variabilis - a. habit; b. flower at anthesis with bracteoles at base; c. stamens of both cycles in lateral view (Burnet (RB 35219)). d-f. Marcetia taxifolia - d. habit; e. flower at anthesis with bracteoles at base; f. stamens of both cycles in lateral view (J.F.A. Baumgratz 799). g-k. Pterolepis glomerata-g. habit; h. flower at anthesis with bracteoles at base; i. fruit covered by branched vascular projections; j. stamens of both cycles in lateral view; k. ovary without style and with apex covered by trichomes (S. Araújo 493). 
3. Marcetia DC., Prodr. 3: 124-125. 1828.

Arbustos e subarbustos; ramos quadrangulares ou subcilíndricos. Folhas planas ou com margens revolutas. Flores 4-meras; estames 8, isomorfos ou subisomorfos, anteras linear-oblongas, com conectivos curtamente prolongados abaixo das tecas e com apêndices ventrais; ovário súpero, 3-4-locular, glabro. Fruto capsular recoberto pelo hipanto persistente.

Marcetia pode ser caracterizado por suas flores tetrâmeras, estames isomorfos a subisomorfos, conectivos curtamente prolongados e inapendiculado ou com apêndices pouco conspícuos (Martins 1989).

São registradas atualmente 31 espécies de Marcetia, todas ocorrentes no Brasil (Santos et al. 2013). A maioria delas é endêmica e com ocorrência restrita aos campos rupestres da Bahia, Minas Gerais e Goiás (Santos et al. 2013). Apenas Marcetia taxifolia (A. St.-Hil.) DC. ocorre fora do território Brasileiro, na Colômbia, Guiana e Venezuela (Martins 2000). Cogniaux (1885) publicou Marcetia glazioviana Cogn. registrada para os estados do Rio de Janeiro, pela coleta de A.F.M. Glaziou de número 8686 (C, K), e Bahia. Porém, Martins (1989) considerou $M$. glazioviana um sinônimo de $M$. taxifolia, com o que concordamos.

3.1 Marcetia taxifolia (A.-St. Hil.) DC., Prodr. 3: 124. 1828. - Rhexia taxifolia A.-St. Hil., in Humboldt \& Bonpland (eds). Monographie des Mélastomacées. Rhexies 2: 150. 1823.

Fig.2d-f

Arbustos ou subarbustos $0,5-1,5 \mathrm{~m}$ alt.; ramos obtuso-tetragonais, não alados, indumento dos ramos, pecíolos e eixos das inflorescências variável, geralmente pubérulo, glandular ou não. Folhas sésseis ou com pecíolos até. $0,5 \mathrm{~mm}$ compr.; lâmina $0,4-0,5 \times 0,1-0,3 \mathrm{~cm}$, oblongo-lanceolada, oval-cordiforme a linear, base cordada a ovada, ápice arredondado ou apiculado, margem revoluta, inconspícuamente serreada e ciliado-glandulosa, ambas as faces hirsuto-glandulosas, (1-)3 nervuras basais, com o par externo inconspícuo. Flores isoladas axilares e no ápice dos ramos; brácteas e bractéolas 1-3 mm compr., lineares. Flores 4-meras, hipanto 2-4 mm compr., oblongocampanulado, hirsuto glandular, lacínias do cálice linear-triangulares, ápice agudo; pétalas ca. 5-7 × 3-4 mm, lilases, purpúreas, róseas ou brancas, ovais; estames 8 , pedoconectivo ausente, subisomorfos, anteras levemente arqueadas ou eretas, linear-oblongas, antepétalos com filetes 5-7 $\mathrm{mm}$ compr., glabros, conectivo com duas aurículas adnadas à base das tecas ca. $0,2 \mathrm{~mm}$ compr., anteras ca. $3 \mathrm{~mm}$ compr.; antessépalos com filetes ca. $4 \mathrm{~mm}$ compr., glabros, conectivos sem aurículas na base das tecas, anteras ca. $4 \mathrm{~mm}$ compr.; ovário glabro, estilete ca. 8-12 mm compr. Cápsula loculicida ca. 3-4 mm compr.

Material selecionado: Araruama, 3.II.1986, fl. e fr., $A$. Costa 3 (RB). Arraial do Cabo, 11.VII.2005, fl. e fr., V.S. Fonseca-Kruel 640 (RB). Cabo Frio, 16.X.1938, fl. e fr., Markgraf, 3041 (RB). Carapebus, 18.X.2007, fl. e fr., $N$. Marquete 617 (RB). Macaé, fl. e fr., 19.XI.2005, J.E. Meireles 399 (RB). Macaé de Cima, 14.IV.2010, fl. e fr., L.A.F. Santos-Filho 153 (RB). Mangaratiba, 5.IV.2008, fl. e fr., K.C. Silva 34 (RB). Nova Friburgo, VII. 1946, fl., J.E. Leite 4093 (RB). Petrópolis, 5.VIII.1983, G. Martinelli 9580 (RB). Quissamã, 11.IX.2010, fl., M.F.O. Silva 701 (R, RFA). Rio das Ostras, 13.VIII.2001, fl., J.F.A. Baumgratz 799 (RB). Rio de Janeiro, 7.XII.1975, fl. e fr., T.C. Pires 2 (RB). Santa Maria Madalena, 25.VI.1987, fl. e fr., C. Farney 1433 (RB). Saquarema, 2.VIII.1994, fl., L.C. Giordano 1682 (RB). Teresópolis, 29.VI.2010, fl. e fr., B. De-Polli et al. (RB 502558).

Marcetia taxifolia é uma espécie de ampla distribuição, ocorrendo também na Venezuela, Guiana e Colômbia. No Brasil ocorre nos estados de Roraima, Alagoas, Bahia, Ceará, Paraíba, Pernambuco, Sergipe, Distrito Federal, Espírito Santo, Minas Gerais, Rio de Janeiro, São Paulo e Paraná (Meyer \& Goldenberg 2012; Martins \& Rodrigues 2014). No estado do Rio de Janeiro pode ser encontrada nos campos de altitude localizados nas porções altas da Serra do Mar, e também em áreas de restinga, na planície litorânea do estado, geralmente em ambientes associados com água.

Marcetia taxifolia é reconhecível pelo hábito arbustivo, folhas diminutas, com margem revoluta, recobertas por tricomas glandulares, e flores tetrâmeras, com anteras lineares com ápice atenuado a subulado. Nesta espécie a morfologia da lâmina foliar é bastante variável, os espécimes coletados em restinga tendem a apresentar folhas com menor dimensão, mais estreitas.

No estado do Rio de Janeiro é relatada a ocorrência de Marcetia bracteolaris (Schrank \& Mart. ex DC.) O. Berg ex Cogn. e Marcetia taxifolia (Martins 1989; Martins \& Rodrigues 2014). Entretanto, Marcetia bracteolaris é uma espécie que está representada apenas pelo material tipo, cuja localidade de coleta é duvidosa, "Minas Gerais" ou "Serra de Sincorá", uma vez que possui duas etiquetas diferentes (Martins 1989). A espécie foi depois referenciada para o "Corcovado, Rio 
de Janeiro" por Cogniaux (1885), que não cita o material examinado proveniente desta localidade. Como esta espécie não foi recoletada no Corcovado desde a descrição, a sua provável ocorrência no estado foi considerada um equívoco de $A$. Cogniaux e por isso não foi tratada neste estudo.

Marcetia taxifolia foi coletada no estado do Rio de Janeiro com flores e frutos ao longo de todos os meses do ano. O status de conservação é pouco preocupante (LC), por ser uma espécie de ampla distribuição e que possui um número significativo de coletas, várias delas recentes.

4. Pterolepis (DC.) Miq., Comm. Phytogr. 2: 72. 1840, nom. cons.

Ervas ou subarbustos; ramos obtusotetragonais a quadrangulares. Folhas planas. Flores 4-5-meras, hipanto com projeções vascularizadas e ramificadas, estames $8-10$, subisomorfos ou dimorfos, anteras subuladas ou truncadas, conectivos prolongados abaixo das tecas com apêndices ventrais bilobados; ovário súpero, 4-5 locular, ápice setoso. Fruto capsular.

Pterolepis é distinto dos demais gêneros de Melastomeae no estado pela presença de projeções vascularizadas e ramificadas recobrindo o hipanto (Meyer \& Goldenberg 2012; Renner 1994). Pterolepis inclui 14 espécies com distribuição geográfica predominante na região central do Brasil. Dentre as que possuem distribuição mais ampla, Pterolepis glomerata (Rottb.) Miq. ocorre nas Américas Central e do Sul, Pterolepis trichotoma (Rottb.) Cogn. no México, em todos os países da América Central, Colômbia, Equador, Venezuela, Guianas, Brasil e Bolívia, Pterolepis repanda (DC.) Triana, ocorre no Brasil e também no Paraguai e Bolívia e Pterolepis stenophylla Gleason não ocorre no território brasileiro, sendo registrada para México, Belize, Guatemala, Honduras, Nicaragua e El Salvador (Renner 1994). A distribuição geográfica de Pterolepis glomerata é tratada nos comentários da espécie (Renner 1994).

4.1 Pterolepis glomerata (Rottb.) Miq., Comm. phytogr. 2: 78. [Feb 1839] 1840. Rhexia glomerata Rottb., Descr. rar. pl. surin.: 8. $1776 . \quad$ Fig 2g-k

Subarbustos 30-60 cm alt.; ramos quadrangulares, não alados, indumento dos ramos, pecíolos e eixos das inflorescências adpressoestrigoso. Folhas sésseis ou com pecíolos ca. 1 mm compr.; lâmina 1,5-3 ×0,5-1 cm, lanceolada, base arredondada a cordada, ápice agudo, margem plana, inteira, face adaxial estrigosa, face abaxial densamente setosa, nervuras 3-basais. Flores isoladas axilares e em inflorescências glomeruliformes no ápice dos ramos; brácteas e bractéolas 3-5 $\mathrm{mm}$ compr., foliáceas. Flores 4-meras, hipanto ca. 3-5 mm compr., campanulado, revestido por projeções vascularizadas e ramificadas, lacínias do cálice triangulares, ápice agudo; pétalas ca. 10-12 × 6-9 mm, magenta, obovadas; estames 8 , subisomorfos, com filetes 4,5-6 mm compr., glabros, pedoconectivos 0,2-0,5 $\mathrm{mm}$ compr., apêndice ventral bilobado, anteras 4,5-5,5 mm compr., falciformes, subuladas; ovário setoso-glandular no ápice, estilete ca. $7 \mathrm{~mm}$ compr. Velatídio ca. $5 \mathrm{~mm}$ compr.

Material selecionado: Angra dos Reis, 12.IV.1969, fl. e fr., D. Sucre 4911 (RB). Arraial do Cabo, X.1961, fl. e fr., A.P. Duarte 5774 (RB). Barra de S. João e Campos Novos, fl. e fr., 25.V.1946, S. Araújo 493 (RB). Cabo, Frio Restinga de Cabo Frio, 9.X. 1968, fl. e fr., D. Sucre 3861 (RB). Carapebus, 21.IV.1995, fl. e fr., P.C.A. Fevereiro 190 (RB). Macaé, 16.VII.1993, fl. e fr., M.G. Bovini 134 (RB). Maricá, 14.X.1979, fl. e fr., H.C. Lima 1108 (RB). Petrópolis, 22.VII.1970, fl. e fr., P. Carauta 1121 (RB). Quissamã, 11.IX.2010, fl. e fr., M.F.O. Silva 703 (R, RFA). Rio de Janeiro, 29.IV.1948, fl. e fr., A.P. Duarte 1167 (RB). Saquarema, 7.III.1989, fl. e fr., A.M.A. Amorim 58 (RB). Teresópolis, 27.IV.1977, G. Martinelli 1816 (RB).

Pterolepis glomerata distribui-se pela República Dominicana, Porto Rico, Antilhas, Venezuela, Guianas, Bolívia, Paraguai e Brasil (Renner 1994) e neste último, em praticamente todos os estados com exceção do Acre, Alagoas, Mato Grosso do Sul, Paraíba e Rio Grande do Sul (Romero 2014b). No estado do Rio de Janeiro ocorre geralmente em restingas, mas também em floresta ombrófila densa na região serrana.

No estado do Rio de Janeiro é a única espécie pertencente à tribo Melastomeae com projeções vascularizadas e ramificadas que recobrem o hipanto (Fig. 2i), sendo assim facilmente reconhecível. Floresce e frutifica ao longo de todos os meses do ano.

Pterolepis glomerata assemelha-se às espécies de Tibouchina que possuem porte herbáceo e frutos com as lacínias persistentes (Meyer \& Goldenberg 2012). Estas pertencentes ao grupo que será sinonimizado em Chaetogastra, e diferem do gênero Pterolepis pela ausência de projeções vascularizadas e ramificadas no hipanto.

No estado do Rio de Janeiro são relacionadas Pterolepis glomerata e Pterolepis trichotoma (Rottb.) Cogn. (Renner 1994; Romero 2014b). 
Contudo a ocorrência de P. trichotoma para o estado é baseada na coleção A.F.M. Glaziou 11956 (K, P) proveniente de "São João da Barra, near Campos". Como a espécie não foi recoletada no estado e as etiquetas das coleções de A.F.M. Glaziou muitas vezes apresentam informações duvidosas como já mencionado (Wurdack 1970), optou-se pela não inclusão deste táxon no trabalho.

Pterolepis glomerata foi coletada com flores e frutos em março, abril, maio, julho, setembro e outubro. O status de conservação é pouco preocupante (LC), por ser uma espécie de ampla distribuição e que possui um número significativo de coletas, várias delas recentes.

\section{Agradecimentos}

Agradecemos ao convênio CAPES-JBRJ e à Secretaria de Educação do Estado do Rio de Janeiro, respectivamente, a bolsa de doutorado e a licença para estudos concedidas à Marcus Felippe Oliveira da Silva; ao Conselho Nacional de Desenvolvimento Científico e Tecnológico (CNPq), o suporte financeiro (REFLORA CNPq, auxílio 563541/2010-5). Aos curadores e funcionários dos herbários visitados e ao ilustrador Natanael Nascimento. A Diana Romano, a revisão do Abstract e das legendas em inglês. Aos revisores, cujas considerações auxiliaram muito na melhoria do texto.

\section{Referências}

Almeda, F. 2009. Melastomataceae. In: Davidse, G.; Sousa-Sánchez; M., Knapp, S. \& Chiang, F. (eds.). Flora mesoamericana. Vol. 4. Universidad Nacional Autónoma de México, México City. Pp. 164-338.

Almeda, F. \& Robinson, O.R. 2011. Systematics and phylogeny of Siphanthera (Melastomataceae). Systematic Botany Monographs 93: 1-101.

Aranha Filho, J.L.M. 2011. Revisão taxonômica das espécies sul-americanas de Symplocos Jacq. seção Hopea (L.) A. DC. (Symplocaceae). Tese de Doutorado. Universidade Estadual de Campinas, São Paulo. 145p.

Barberena, F.F.V.; Baumgratz, J.F.A. \& Chiavegatto, B. 2008. Melastomataceae no Parque Nacional do Itatiaia, Sudeste do Brasil: Tribos Bertolonieae e Merianieae. Rodriguésia 59: 381-392.

Baumgratz, J.F.A. 1985. Morfologia dos frutos e sementes de melastomatáceas brasileiras. Arquivos do Jardim Botânico do Rio de Janeiro 27: 113-155.

Baumgratz, J.F.A. 2014a. Appendicularia DC. In: Lista de espécies da flora do Brasil. Jardim Botânico do Rio de Janeiro. Disponível em $<$ http://floradobrasil.
jbrj.gov.br/jabot/floradobrasil/FB85199>. Acesso em 22 julho 2014.

Baumgratz, J.F.A. 2014b. Comolia DC. In: Lista de espécies da flora do Brasil. Jardim Botânico do Rio de Janeiro. Disponível em < http://floradobrasil.jbrj. gov.br/jabot/floradobrasil/FB9453>. Acesso em 22 julho 2014.

Baumgratz, J.F.A. 2014c. Comoliopsis Wurdack. In: Lista de espécies da flora do Brasil. Jardim Botânico do Rio de Janeiro. Disponível em $<$ http://floradobrasil. jbrj.gov.br/jabot/floradobrasil/FB111278>. Acesso em 22 julho 2014.

Baumgratz, J.F.A. 2014d. Desmoscelis. Naudin. In: Lista de espécies da flora do Brasil. Jardim Botânico do Rio de Janeiro. Disponível em $<$ http://floradobrasil. jbrj.gov.br/jabot/floradobrasil/FB9459>. Acesso em 22 julho 2014.

Baumgratz, J.F.A. 2014e. Ernestia DC. In: Lista de espécies da flora do Brasil. Jardim Botânico do Rio de Janeiro. Disponível em <http://floradobrasil.jbrj. gov.br/jabot/floradobrasil/FB79261>. Acesso em 22 julho 2014.

Baumgratz, J.F.A. 2014f. Fritzschia Cham. In: Lista de espécies da flora do Brasil. Jardim Botânico do Rio de Janeiro. Disponível em < http://floradobrasil.jbrj. gov.br/jabot/floradobrasil/FB9467> . Acesso em 22 julho 2014.

Baumgratz, J.F.A. 2014g. Itatiaia Ule. In: Lista de espécies da flora do Brasil. Jardim Botânico do Rio de Janeiro. Disponível em < http://floradobrasil.jbrj. gov.br/jabot/floradobrasil/FB9490>. Acesso em 22 julho 2014.

Baumgratz, J.F.A. 2014h. Loricalepis Brade. In: Lista de espécies da flora do Brasil. Jardim Botânico do Rio de Janeiro. Disponível em $<$ http://floradobrasil. jbrj.gov.br/jabot/floradobrasil/FB79270>. Acesso em 22 julho 2014.

Baumgratz, J.F.A. 2014i. Macairea DC. In: Lista de espécies da flora do Brasil. Jardim Botânico do Rio de Janeiro. Disponível em < http://floradobrasil.jbrj. gov.br/jabot/floradobrasil/FB19632>. Acesso em 22 julho 2014.

Baumgratz, J.F.A. 2014j. Nepsera Naudin. In: Lista de espécies da flora do Brasil. Jardim Botânico do Rio de Janeiro. Disponível em <http://floradobrasil.jbrj. gov.br/jabot/floradobrasil/FB25810>. Acesso em 22 julho 2014.

Baumgratz, J.F.A.2014k. Poteranthera Bong. In: Lista de espécies da flora do Brasil. Jardim Botânico do Rio de Janeiro. Disponível em < http://floradobrasil. jbrj.gov.br/jabot/floradobrasil/FB9826>. Acesso em 22 julho 2014.

Baumgratz, J.F.A. 20141. Tibouchinopsis Markgr. In: Lista de espécies da flora do Brasil. Jardim Botânico do Rio de Janeiro. Disponível em $<$ http://floradobrasil.jbrj.gov.br/jabot/floradobrasil/ FB30800>. Acesso em 22 julho 2014. 
Baumgratz, J.F.A. \& Lima, L.F.G. 2014. Sandemania Gleason. In: Lista de espécies da flora do Brasil. Jardim Botânico do Rio de Janeiro. Disponível em $<$ http://floradobrasil.jbrj.gov.br/jabot/floradobrasil/ FB23770>. Acesso em 22 julho 2014.

Baumgratz, J.F.A. \& Rosa, P. 2014. Aciotis D. Don. In: Lista de espécies da flora do Brasil. Jardim Botânico do Rio de Janeiro. Disponível em $<$ http://floradobrasil.jbrj.gov.br/jabot/floradobrasil/ FB9389>. Acesso em 24 fevereiro 2014.

Baumgratz, J.F.A. \& Souza, M.L.D.R. 2011. Melastomataceae na Reserva Ecológica de Macaé de Cima, Nova Friburgo, Rio de Janeiro, Brasil. II - Leandra (Miconieae). Rodriguésia 62: 629-662.

Baumgratz, J.F.A.; Souza, M.L.D.R.; Carraça, D.C. \& Abbas, B.A. 2006. Melastomataceae na Reserva Biológica de Poço das Antas, Silva Jardim, RJ: aspectos florísticos e taxonômicos. Rodriguésia 57: 591-646.

Baumgratz, J.F.A.; Souza, M.L.D.R. \& Tavares, R.A.M. 2004. Bisglaziovia Cogn. (Merianieae, Melastomataceae): Considerações Taxonômicas e Biogeográficas. Bradea 10: 75-80.

Baumgratz, J.F.A.; Souza, M.L.D.R. \& Tavares, R.A.M. 2007. Melastomataceae na Reserva Ecológica de Macaé de Cima, Nova Friburgo, Rio de Janeiro, Brasil I - Tribos Bertolonieae, Merianieae e Microlicieae. Rodriguésia 58: 797-822.

Baumgratz, J.F.A.; Rodrigues, K.F.; Chiavegatto, B.; Goldenberg, R.; Guimarães, P.J.F.; Kriebel, R.; Martins, A.B.; Michelangeli, F.A.; Reginato, M.; Romero, R.; Souza, M.L.D.R.; Woodgyer, E.; Caddah, M.K.; Koschnitzke, C.; Lima, L.F.G. \& Rosa, P. 2014. Melastomataceae In: Lista de espécies da flora do Brasil. Jardim Botânico do Rio de Janeiro. Disponível em < http://floradobrasil.jbrj. gov.br/jabot/floradobrasil/FB161>. Acesso em 22 fevereiro 2014.

Barroso, G. M. (1958). Mikanieae do Brasil. Arquivos do Jardim Botânico do Rio de Janeiro 16: 322.

Berry, P.E.; Gröger, A.; Holst, B.K.; Morley, T.; Michelangeli, F.A.; Luckana, N.G.; Almeda, F.; Renner, S.S.; Freire-Fierro, A.; Robinson, O.R. \& Yatskievych, K. 2001. Melastomataceae. In: Berry, P.E., Yatskievych, K. \& Holst, B.K. Flora of the Venezuelan Guayana. Missouri Botanical Garden Press, St. Louis. Pp 387-468.

Chiavegatto, B. \& Baumgratz, J.F.A. 2008. Meriania (Melastomataceae; Merianieae) no Rio de Janeiro, Brasil. Rodriguésia 59: 899-913.

Clausing, G. \& Renner, S.S. 2001. Molecular phylogenetics of Melastomataceae and Memecylaceae: implications for character evolution. American Journal of Botany 88: 486-498.

Cogniaux, A. 1885. Marcetia DC. (Melastomataceae). In: C.F.P. Martius, A.G. Eichler \& I. Urban (eds.). Flora brasiliensis. Leipzig, Vol. 14, pp. 288-417.
Freire-Fierro, A. 2002. Monograph of Aciotis (Melastomataceae). Systematic Botany Monographs 62: 1-99.

Goldenberg, R. 2014. Monochaetum. (DC.) Naudin. In: Lista de espécies da flora do Brasil. Jardim Botânico do Rio de Janeiro. Disponível em $<$ http://floradobrasil.jbrj.gov.br/jabot/floradobrasil/ FB130782>. Acesso em 22 julho 2014.

Goldenberg, R.; Baumgratz, J.F.A. \& Souza, M.L.D.R. 2012. Taxonomia de Melastomataceae no Brasil: retrospectiva, perspectivas e chave de identificação para os gêneros. Rodriguésia 63: 145-161.

Guimarães, P.J.F. 2009. Acisanthera P. Browne. In: Wanderley, M.G.L.; Shepherd, G.J.; Melhem, T.S.; Giulietti, A.M. \& Martins, S.E. (eds.). Flora fanerogâmica do estado de São Paulo. Vol. 6. FAPESP, São Paulo. Pp 5-7.

Guimarães, P.J.F. 2014. Tibouchina Aubl. In: Lista de espécies da flora do Brasil. Jardim Botânico do Rio de Janeiro. Disponível em < http://floradobrasil.jbrj. gov.br/jabot/floradobrasil/FB9876>. Acesso em 22 julho 2014

Hickey, M. \& King, C. 2000. The Cambridge illustrated glossary of botanical terms. Cambridge University Press, Cambridge. 208p.

Jstor. 2014. Schwacke, Carl August Wilhelm (18481904). Disponível em $<\mathrm{http}$ ://plants.jstor.org/person/ bm000007632?s=t.> . Acesso em 6 agosto 2014.

Kriebel, R. 2008. Systematics and biogeography of the Neotropical genus Acisanthera (Melastomataceae). MSc thesis. Faculty of San Francisco State University, San Francisco. 175p.

Kriebel, R. 2014. Acisanthera P.Browne In: Lista de espécies da flora do Brasil. Jardim Botânico do Rio de Janeiro. Disponível em < http://floradobrasil.jbrj. gov.br/jabot/floradobrasil/FB9395> . Acesso em 24 fevereiro 2014.

Kriebel, R. \& Almeda, F. 2013. Clinal variation and the decoupling of vegetative and reproductive characters in Acisanthera section Acisanthera (Melastomataceae). Harvard Papers in Botany 18: 157-172.

Martins, A.B. 1989. Revisão taxonômica do gênero Marcetia DC. (Melastomataceae). Tese de doutorado. Universidade Estadual de Campinas. Campinas. 277p.

Martins, A.B. 2000. Three new brazilian species in the genus Marcetia (Melastomataceae, Melastomeae). Novon 10: 224-229.

Martins, A.B. \& Rodrigues, K.F. 2014. Marcetia DC. In: Lista de espécies da flora do Brasil. Jardim Botânico do Rio de Janeiro. Disponível em $<$ http://floradobrasil.jbrj.gov.br/jabot/floradobrasil/ FB9646>. Acesso em 24 fevereiro 2014.

Mendoza, H. \& Ramírez, B. 2006. Guía ilustrada de géneros de Melastomataceae y Memecylaceae de Colombia. Instituto de Investigación de Recursos Biológicos 
Alexander von Humboldt; Universidad del Cauca, Bogotá. 288p.

Meyer, F.S. \& Goldenberg, R. 2012. Aciotis, Acisanthera, Marcetia, Microlepis, Pterolepis e Siphanthera (Melastomataceae, Melastomeae) no Estado do Paraná, Brasil. Rodriguésia 63: 293-303.

Michelangeli, F. A. \& Cotton, E. 2008. Melastomataceae. In: Hokche, R.; Berry, P.E. \& Huber, O. (eds.). Nuevo catalogo de la flora vascular de Venezuela. Fundación Instituto Botánico de Venezuela Dr. Tobías Lasser, Caracas. Pp. 466-484.

Michelangeli, F.A.; Guimarães, P.J.F.; Penneys, D.S.; Almeda, F. \& Kriebel, R. 2013. Phylogenetic relationships and distribution of new world Melastomeae (Melastomataceae). Botanical Journal of the Linnean Society 171: 38-60.

Oliveira da Silva, M.F., Guimarães, P.J.F. \& Michelangeli, F.A. 2014. Nomenclatural and taxonomic novelties in the tribe Melastomeae (Melastomataceae). Phytotaxa 186: 222-228.

Penneys, D.S.; Michelangeli, F.A.; Judd, W.S. \& Almeda, F. 2010 Henrietteeae (Melastomataceae): A new neotropical berry-fruited tribe. Systematic Botany 35: 783-800.

Peralta, P. 2002. Las especies del género Tibouchina (Melastomataceae) en Argentina. Darwiniana. 40: 107-120.

Pereira, E. 1960-1961. Flora do estado da Guanabara III. Melastomataceae I - Tibouchineae. Rodriguésia 23-24: 155-188.

Radford, A.E.; Dickinson, W.C.; Massey, J.R. \& Bell, C.R. 1974. Vascular plant systematics. Harper \& Row Publishers, New York. 891p.

Renner, S.S. 1993. Phylogeny and classification of the Melastomataceae and Memecylaceae. Nordic Journal of Botany 13: 519-540.

Renner, S.S. 1994. A revision of Pterolepis (Melastomataceae: Melastomeae). Nordic Journal of Botany 14: 73-104.

Romero, R. 2014a. Microlepis (DC.) Miq. In: Lista de Espécies da Flora do Brasil. Jardim Botânico do Rio de Janeiro. Disponível em $<$ http://floradobrasil. jbrj.gov.br/jabot/floradobrasil/FB9780>. Acesso em 22 julho 2014
Romero, R. 2014b. Pterolepis (DC.) Miq. In: Lista de espécies da flora do Brasil. Jardim Botânico do Rio de Janeiro. Disponível em <http://floradobrasil.jbrj. gov.br/jabot/floradobrasil/FB9856>. Acesso em 24 fevereiro 2014.

Romero, R. 2014c. Siphanthera. Pohl. ex DC. In: Lista de espécies da flora do Brasil. Jardim Botânico do Rio de Janeiro. Disponível em $<$ http://floradobrasil. jbrj.gov.br/jabot/floradobrasil/FB9870>. Acesso em 22 julho 2014

Romero, R. 2014d. Svitramia. Cham. In: Lista de espécies da flora do Brasil. Jardim Botânico do Rio de Janeiro. Disponível em <http://floradobrasil.jbrj. gov.br/jabot/floradobrasil/FB30824>. Acesso em 22 julho 2014.

Romero, R. \& Versiane, A.F.A. 2014. Taxonomic Novelty and Typifications in Microlepis (Melastomataceae). Novon 2: 217-223.

Santos, A.K.A.; Martins, A.B. \& Silva, T.S. 2013. Two New Species of Marcetia (Melastomataceae) from the Chapada Diamantina, Bahia, Brazil. Systematic Botany 38: 714-722.

Silva, K. C. \& Baumgratz, J. F. A. 2008. Henriettea e Henriettella (Melastomataceae) no estado do Rio de Janeiro, Brasil. Rodriguésia 59: 887-897.

Silva, M.F.O.; Andreata, R.H.P. \& Guimarães, P.J.F. 2013. Melastomataceae no Parque Estadual da Pedra Branca, Rio de Janeiro, RJ, Brasil. Hoehnea 40: 679-700.

Thiers, B. 2014. [continuously updated]. Index herbariorum: a global directory of public herbaria and associated staff. New York Botanical Garden's Virtual Herbarium. Disponível em $<$ http://sweetgum. nybg.org/ih/>. Acesso em 22 fevereiro 2014.

Veloso, H.P.; Filho, A.L.R.R. \& Lima, J.C.A. 1991. Classificação da vegetação brasileira adaptada a um sistema universal. Instituto Brasileiro de Geografia e Estatística, Rio de Janeiro. 124p.

Whiffin, T. \& Tomb, A.S. 1972. The systematic significance of seed morphology in the Neotropical capsular-fruited Melastomataceae. American Journal of Botany 59: 411-422.

Wurdack, J.J. 1970. Erroneous data in Glaziou collections of Melastomataceae. Taxon 19: 911-913. 
Lista de exsicatas

Almeida, G.F. RB238807 (4); Amorim, A.M.A. 58(4); Araujo, S. 485(4), 493(4); Baumgratz, J.F.A. 799(4), 803(4), 876(1); Barros, W.D. 561(2); Braga, P.I.S. 2371(4); Bovini, M.G. 134(4), 154(4), 783(4), 2217(1), 2112(2); Brade, A.C. 12751(4), 14259(4), 15787(4), 15788(4), 16890(4), 18665(4); Braga, J.M.A. 1900(1); Burnet. RB35219(2); Carauta, J.P.P. 425(4), 1121(4), 1614(4); Carvalho, L.A.F. 13(4); Castro, M.B. 2(4); Costa, A. 3(4); De-Polli, B. RB502558(3), 18(1); Dionísio. RB10801(1); Duarte, A. 1167(4), 5760(4), 5774(4), 5857(4); Emygdio, L. 3086(1); Farney, C. 330(4), 596(4), 1433(4), 3416(4), 3508(4); Fevereiro, P.C.A. 9(4), 104(4), 190(4); Fonseca-Kruel, V.S. 640(4); Fontela, J. 2277(4), 3111(4); Forzza, R.C. 2898(4); Fromm, E. 1256(1); Giordano, L.C. 1682(4); Glaziou, A.F.M. 332(4), 8686(3), 10769(2), 12686(3), 16814(4); Goes, O.C. 9(2), 120(2), 243(2), 801(4); Hunt, D.R. 5427(4); Ichaso, C.L.F. 189(4); Laboriau, A. 290(4); Lobão, A. 7(4); Kirkbride, J.H. RB237945(4); Lanstyack, L. 286(2), 302(2); Leite, J.E. 4093(4); Lemos, M.H.O.15(4), 33(4); Liane, D. 3522(4), 3738(4); Lima, H.C. 658(4), 673(4), 1108(4), 4631(1); Lima, S. 355(4); Lutz, B. 1563(1); Maas, P.J.M. 3143(4); Markgraf, F. 3041(4); Marquete, N. 617(4), 641(4); Martinelli, G. 1816(4), 9324(4), 9580(4); Meireles, J.E. 399(4); Menezes, L.F.T. 252(4); Pabst, G.F.J. RB77123(4); Paixão, R. 323(4); Peixoto, S.L. RB237929(4); Pereira, E. 629(1); Pilger. RB258155(2); Pirani, J.R. 2868(3); Piratininga, A. 17(1); Pires, T.C. 2(3); Poland, C. 6664(3); Sampaio, A. 1612(2); Santos, M.G. 316(3), 512(4); Santos-Filho, L.A.F. 153(3); Santos-Lima, J. 14258(3); Schwacke, P. 1190(4), 1503(3), 5568(4); Silva, K.C. 34(3); Silva, M.F.O. 701(3), 703(4); Silva-Neto, S.J. 1610(1), 1609(2); Siqueira, M.J.N. 1(3); Sucre, D. 1055(3), 2740(2), 3843(3), 3861(4), 4491(2), 4911(4), 5037(3), 5727(3), 9173(3); Ule, E. R166536(1); Vianna, M.C. 553(3); Wendt, T. 14(3). 
